\title{
Identification of the Gene Encoding the Major Latency-associated Nuclear Antigen of the Kaposi's Sarcoma-associated Herpesvirus
}

\author{
Dean H. Kedes, ${ }^{\star}$ Michael Lagunoff, ${ }^{*}$ Rolf Renne, ${ }^{\star}$ and Don Ganem ${ }^{\star \ddagger \S}$ \\ ${ }^{*}$ Department of Microbiology and Immunology and ${ }^{\ddagger}$ Department of Medicine and the ${ }^{\S}$ Howard Hughes Medical Institute, University of \\ California Medical Center, San Francisco, California 94143-0414
}

\begin{abstract}
Over $85 \%$ of patients with Kaposi's sarcoma (KS) are seropositive for antibodies to the latency-associated nuclear antigen (LANA) expressed in B cell lines infected with Kaposi's sarcoma-associated herpesvirus (KSHV). The presence of antibodies to LANA strongly correlates with the risk of developing the disease. However, the identity of the protein(s) comprising LANA and the corresponding gene(s) has remained unclear. To identify potential latent gene candidates for LANA, we probed total RNA extracted from BCBL-1 cells (a B cell line latently infected with KSHV) using lambda clones that span the KSHV genome. One region encoding latent transcripts spanned KSHV open reading frames (orfs) 71 (K13), 72 (v-cyclin), and 73. Among these, however, only orf 73, when expressed in heterologous mammalian cell systems, reacted with KSHV antibody-positive human sera, resulting in a punctate nuclear staining pattern reminiscent of LANA in BCBL-1 cells. Furthermore, extracts from cells expressing the orf 73 protein product specifically blocked the binding of $\mathrm{KS}$ patient antibodies to LANA. Finally, seroreactivity with recombinant orf 73 protein exactly paralleled reactivity with classical LANA as expressed in BCBL-1 cells, both in KS patients and in other groups. Together, these data support the identification of KSHV orf 73 as the gene encoding the dominant immunogenic component of LANA. (J. Clin. Invest. 1997. 100:26062610.) Key words: HHV8 • KSHV • Kaposi's sarcoma
\end{abstract}

\section{Introduction}

Kaposi's sarcoma-associated herpesvirus (KSHV) ${ }^{1}$ also termed human herpesvirus 8 (HHV8), is implicated as a major etiologic agent in the formation of Kaposi's sarcoma (KS) (1-6),

\footnotetext{
Address correspondence to Don Ganem, Howard Hughes Medical Institute, University of California Medical Center, San Francisco, CA 94143-0414. Phone: 415-476-2826; FAX: 415-476-0939; E-mail: ganem@ socrates.ucsf.edu

Received for publication 10 July 1997 and accepted in revised form 25 September 1997.
}

1. Abbreviations used in this paper: HHV8, human herpesvirus 8; IFA, immunofluorescence assay; KS, Kaposi's sarcoma; KSHV, KSassociated herpesvirus; LANA, latency-associated nuclear antigen; orf, open-reading frame; PEL, primary effusion lymphoma; TPA, 12$O$-tetradecanoylphorbol-13-acetate.

J. Clin. Invest.

(C) The American Society for Clinical Investigation, Inc. 0021-9738/97/11/2606/05 \$2.00

Volume 100, Number 10, November 1997, 2606-2610

http://www.jci.org primary effusion lymphomas (PEL; previously known as body cavity-based lymphomas) (7) and a large fraction of cases of multicentric Castleman's disease (8). Seroepidemiologic and PCR-based studies of patients with these diseases have revealed that viral infection precedes the onset of KS (9-12; D. Kedes and D. Ganem, unpublished findings) and is predictive of an increased risk of tumor development $(9,10,13,14)$. The groups at highest risk of developing KS, namely HIV-positive homosexual men, have the highest KSHV seroprevalence rates (13-16). In contrast, those groups with lower risks of KS development, for example eligible blood donors and even HIV-positive women, intravenous drug users, and hemophiliacs, show correspondingly low rates of seropositivity $(13,14$, 16-18). Furthermore, the major route of transmission in nonendemic countries seems to be sexual $(13,15,16)$ with its prevalence in homosexual men, for example, rising with greater numbers of sexual partners (J. Martin and D. Kedes, unpublished observations), a known risk factor for KS development $(19,20)$.

Many of these serologic studies have been based on the detection of antibodies to a latency-associated nuclear antigen (LANA) that is expressed in B cell lines latently infected with $\mathrm{KSHV}$. Infection with KSHV is specifically associated with the development of antibodies to LANA; immunofluoresence assays using sera from infected individuals give a punctate nuclear distribution of LANA in such lines, which are derived from cases of PEL $(13,14,16)$. These same sera in Western blots of nuclear extracts from these cell lines react with a high molecular weight polypeptide $(11,16$; D. Kedes and D. Ganem, unpublished observations). Seroepidemiologic surveys demonstrate that $>85 \%$ of patients with Kaposi's sarcoma (KS) possess anti-LANA antibodies while only $1-4 \%$ of the sera from the general population are positive for such antibodies (13-16).

While these studies presumptively characterize LANA as a viral antigen expressed during the latent phase of infection, they do not specifically identify the viral gene product(s) detected. Since latently expressed genes in other lymphotropic herpesviruses play critical roles in the biology of infection (21), the identification of cognate genes in KSHV is likely to be important in developing a better understanding of the interaction of this virus with its host. In addition, the cloning of the genes for such antigens makes possible the development of secondgeneration serologic tests for KSHV infection based upon defined recombinant antigens. Here we identify the major component of LANA as the product of the viral open-reading frame (orf) 73.

\section{Methods}

Cell culture. COS7, 293 and CV-1 cells were cultured in DME-H16 supplemented with $10 \%$ fetal calf serum (Lifetechnologies, Inc., Gaithersburg, MD), $50 \mu \mathrm{g} / \mathrm{ml}$ penicillin and $50 \mu \mathrm{g} / \mathrm{ml}$ streptomycin. Cells were cultured at $37^{\circ} \mathrm{C}$ in $5 \% \mathrm{CO}_{2}$. BCBL-1 cells were cultured 
as previously described (22). Lytic replication of KSHV in BCBL-1 cells was induced by TPA as described (22).

RNA extraction and Northern blotting. BCBL-1 cells were grown to $3 \times 10^{5}$ cells per $\mathrm{ml}$ and either left uninduced or induced with 12 $O$-tetradecanoylphorbol-13-acetate (TPA; $20 \mathrm{ng} / \mathrm{ml}$ ). $48 \mathrm{~h}$ later total RNA from both sets of cells was harvested using RNAzol as recommended by the manufacturer (Tel-Test, Friendswood, TX). Equivalent amounts of RNA were then polyA enriched using the Oligotex protocol (Qiagen, Chatsworth, CA) and separated on a 1\% agarose/ formaldehyde gel according to standard protocols. The gel was subsequently transferred to hybond- $N$ membrane (Amersham International, Buckinghamshire, United Kingdom) and reacted with the appropriate probe as described previously (22). Probes were made by digesting previously isolated lambda clones that encompass $98 \%$ of the unique region of the genome $(23,24)$ with appropriate restriction enzymes, gel purifying specific bands (between 1 and $9 \mathrm{kbp}$ ) and radiolabeling using the rediprime protocol (Amersham International). Membranes were exposed to XAR5 film overnight (Eastman Kodak Co., Rochester, NY).

Patient sera. Sera collected from KS patients, eligible blood donors, and from women without sexual exposure were described previously (13).

Cloning of orf 73. A DNA fragment that included all of orf 73 was recovered from lambda clone 4 (reference 24) after a KpnI/NheI restriction digest. This fragment was ligated into the mammalian expression vector pcDNA3 (Invitrogen, Carlsbad, CA) linearized with $\mathrm{KpnI} / \mathrm{XbaI}$. The resulting clone is referred to as pcDNA3-orf 73 .

Transfections. COS7 cells in log phase growth were transfected with either pcDNA3 or with pcDNA3-orf 73 using lipofection with LipofectAMINE (Lifetechnologies, Inc.) according to manufacturer's recommendations. Transfection efficiency was evaluated by cotransfecting parallel cultures with either of the pcDNA3-based plasmids plus one-tenth the molarity of the lacZ containing plasmid $\mathrm{pCH} 110$ (Pharmacia Biotechnology, Uppsala, Sweden). $\beta$-galactosidase assays were performed on cells $48 \mathrm{~h}$ after transfection as described previously (25). $10-20 \%$ of the cells were typically positive with this assay.

COS7 cell sonicates. $48 \mathrm{~h}$ after transfection, cells from confluent 10-cm tissue culture dishes were collected by centrifugation at $500 \mathrm{~g}$ for $5 \mathrm{~min}$ and then washed once with PBS. The pellets were then resuspended by gentle vortexing in prechilled sonication buffer $(50 \mathrm{mM}$ Tris-HCl [pH 8.0], $0.5 \mathrm{mM}$ EDTA [pH 8.0], $450 \mathrm{mM} \mathrm{NaCl}, 0.2 \mathrm{mM}$ phenylmethylsulfonyl fluoride, and $0.5 \mathrm{mM}$ DTT). The cell suspension was then sonicated on setting 2 (Sonifer 250; Branson Ultrasonics Corp., Danbury, CT) on ice until nuclei were no longer intact. After insoluble material was removed by centrifugation at $14,000 \mathrm{~g}$ at $4^{\circ} \mathrm{C}$ for $2 \mathrm{~min}$ in a microcentrifuge, the protein concentration of the supernatant was determined using the Bio-Rad protein assay (Hercules, CA) according to the manufacturer's recommendations. Aliquots of the extracts diluted with sonication buffer to a concentration of $20 \%$ (wt $/ \mathrm{vol}$ ) protein were then stored at $-70^{\circ} \mathrm{C}$ until required.

Immunofluorescence assays (IFA). Patient sera were diluted with PBS as indicated in the figure legends and then incubated with COS7 cells or with nuclei isolated from BCBL-1 cells as described previously (13). For adsorption experiments, diluted KS and control sera were incubated overnight at $4^{\circ} \mathrm{C}$ with sonicates from COS7 cells transfected with either pcDNA3 or pCDNA3-orf 73. The final protein concentration in the serum-extract mixes was kept constant $(10 \%, \mathrm{wt} / \mathrm{vol})$. The adsorbed sera were then used in the IFAs with BCBL-1 nuclei. IFAs were visualized by immunofluorescent microscopy (Nikon Microphone-FAX; Nikon Inc., Tokyo, Japan) at a 600fold magnification. Photographs were taken with Kodachrome 400 ASA film (Eastman Kodak Co.).

\section{Results}

The phorbol ester 12-O-tetradecanoylphorbol-13-acetate (TPA) induces a marked increase in the transcription of the lytic genes in cultured cells latently infected by many lymphotropic herpesviruses, including $\operatorname{KSHV}(22,26)$. Without such stimulation, these transcripts are present at very low or neglible levels. In contrast, transcripts of many latent viral genes are expected to be present in the absence of TPA induction. Exploiting this difference, we attempted to identify regions of the KSHV genome encoding potential latent gene products by screening for regions actively transcribed in the absence of TPA. We reasoned that this process would narrow the choices of potential candidate genes that could encode the latency-associated nuclear antigen (LANA) that we and others previously identified by $\operatorname{IFA}(13,14)$.

Accordingly, we prepared RNA from BCBL-1 cells grown in the presence or absence of TPA and examined this RNA for viral gene expression by Northern blotting, using as probes a number of large viral DNA fragments from across the $170-\mathrm{kb}$ viral genome (24). As shown in Fig. 1 (lanes 1 and 2) one clone (lambda 4-3) revealed the presence of viral transcripts that were of nearly equal abundance in both uninduced and induced cultures, strongly suggesting that this clone harbors latently expressed viral genes. DNA sequencing revealed that this clone spans KSHV orfs 71, 72, and 73 (detailed analyses of the transcripts and their promoters from this region will be published elsewhere; Dirk Dittmer and D. Ganem, manuscript in preparation). In contrast, most other clones tested revealed a marked increase in the levels of transcripts, consistent with lytically expressed viral genes. Fig. 1 (lanes 3 and 4) demonstrate an example of this latter pattern with a probe from lambda clone 13-3 (reference 24) encompassing the glycoprotein $\mathrm{M}$ homolog (27). Of course, inducibility by TPA does not preclude the possibility of latent gene expression: for example, any latent gene which continues to be transcribed during the lytic cycle would show transcript accumulation as a result of DNA copy number amplification. It is therefore highly likely that additional latent genes exist outside of the region encompassed by lambda 4-3.

To determine which (if any) of the orfs 71-73 might encode LANA, we then subcloned each of these three orfs into mammalian expression vectors. The resulting clones were then

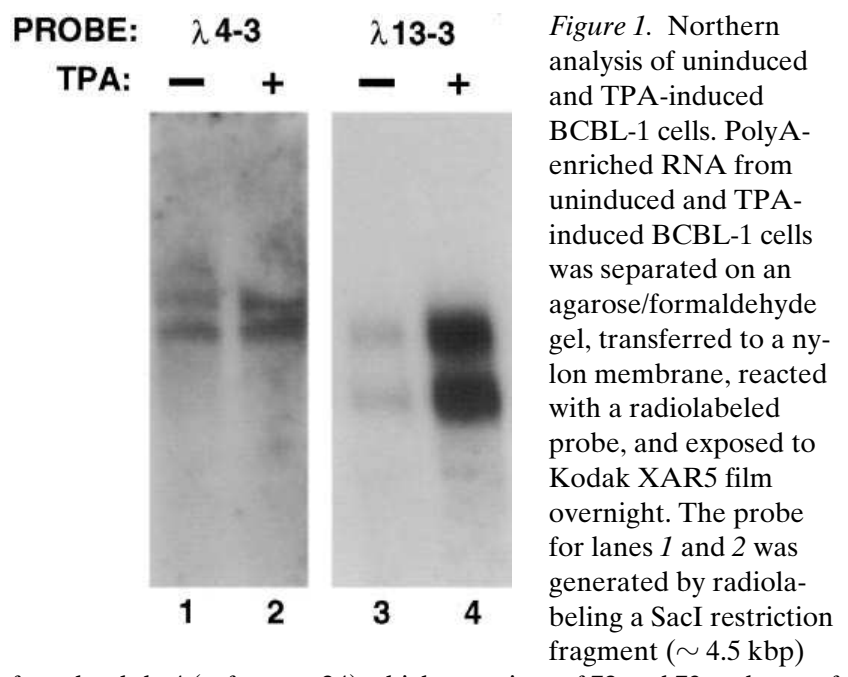

from lambda 4 (reference 24) which contains orf 72 and 73 and part of ORF 71 (K13). The probe for lanes 3 and 4 was generated by radiolabeling the fourth largest SalI fragment $(\sim 2 \mathrm{kbp})$ from lambda 13 (reference 24) which contains the region around orf 38 and 39 (glycoprotein M homolog) (27). 

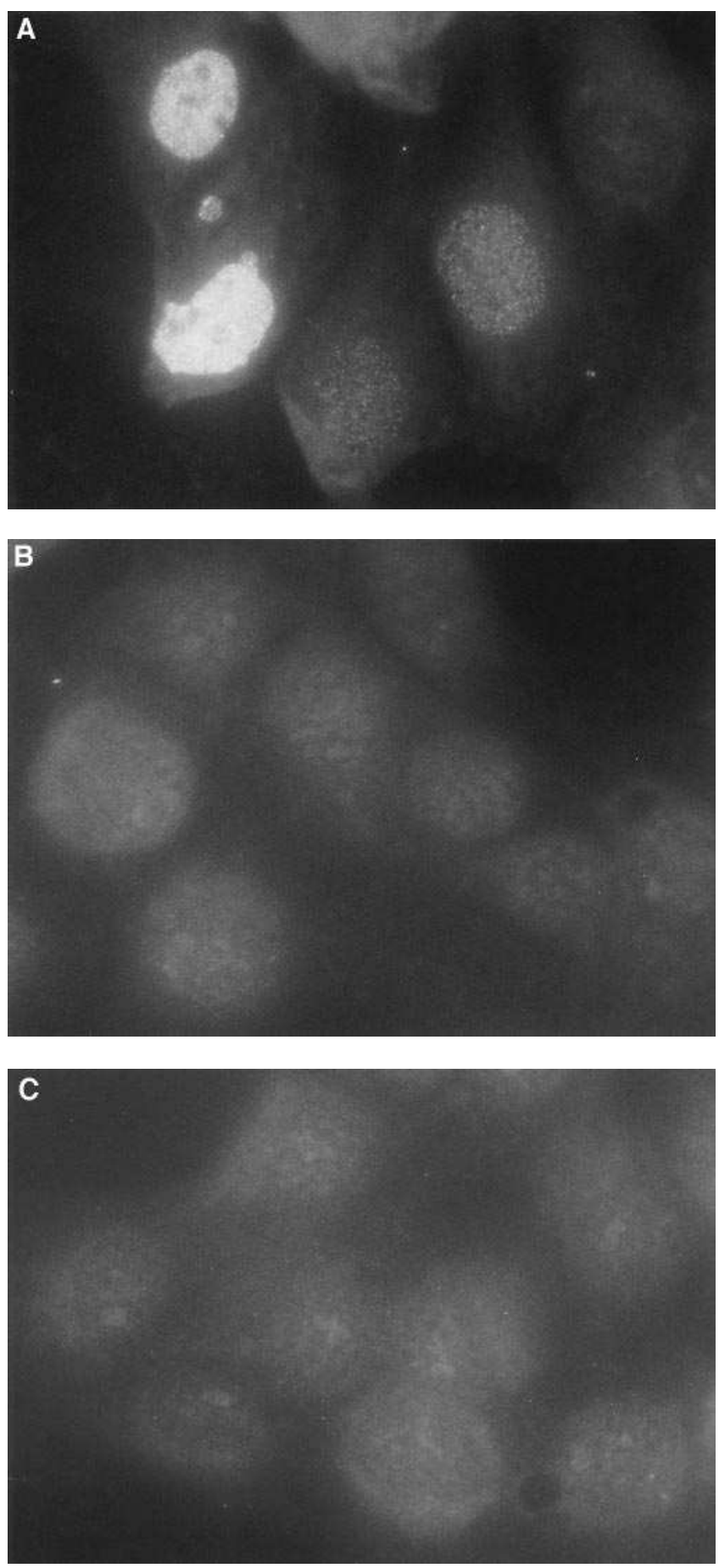

Figure 2. Immunofluorescence pattern of $\operatorname{COS} 7$ cells transfected with pcDNA3-orf 73 and reacted with a 1:100 dilution of serum from either a patient with $\mathrm{KS}(A)$ or a control subject $(B)$. COS7 cells transfected with pcDNA3 reacted with KS patient serum is seen in panel $(C)$.

transfected individually into COS7 cells and, $2 \mathrm{~d}$ later, the monolayers were assayed by immunofluorecence using KS patient antisera known to be positive for anti-LANA antibodies in high titer. Cells transfected with the orf 71 and 72 expression vectors gave no discernible immunofluorescence signal in this assay, despite efficient transfection, as judged by $\beta$-galactosi- dase expression from a cotransfected lacZ reporter gene. By contrast, cells bearing the orf 73 vectors displayed a bright nuclear fluorescence pattern with these same sera. (Recent work in our laboratory has revealed that the orf 73 mRNA is $4.6 \mathrm{~kb}$ in BCBL-1 cells; Dirk Dittmer and D. Gamen, manuscript in preparation.) Although the punctate staining in these COS7 cells revealed a finer pattern than that observed in staining of BCBL-1 cells, it shared the same exclusively nuclear targeting and strong sparing of nucleoli. At least some of the difference in the exact distribution of the staining are likely the result of different levels of protein expression in these cells, since the punctate pattern is more evident in COS7 cells displaying lower levels of fluorescence (see Fig. $2 A$ ). Moreover, the signal was not detected in orf 73-expressing cells reacted with control human sera lacking anti-LANA antibodies (Fig. $2 \mathrm{~B}$ ) or in control cells transfected with pcDNA3 alone (Fig. $2 C$ ). In addition, transfection of 293 and CV-1 cells with the orf 73 construct gave rise to nuclear immunofluorescent patterns very similar to those in the COS7 cells when reacted with LANA antibody positive sera (data not shown).

Although the nuclear pattern was reminiscent of LANA in BCBL-1 cells, it remained formally possible that either (i) orf 73 may merely encode another unrelated nuclear antigen or (ii) orf 73 may comprise only a single component of a multiantigen complex representing LANA. To distinguish between these possibilities, we preadsorbed sera positive for LANA reactivity with COS7 cell sonicates that had been transfected with either pcDNA3 or the orf 73-expressing plasmid. If orf 73 were indeed identical with or a major component of LANA, then such preadsorption should attenuate all or a part of the LANA pattern in IFAs on BCBL-1 nuclei.

Fig. 3 shows the results of such an experiment. The characteristic LANA pattern was undisturbed when KS patient serum was preadsorbed exclusively with sonicate from COS7 cells transfected with the control vector (Fig. $3 A$ ). However, preadsorption with increasing amounts of sonicate from COS7 cells transfected with the orf 73 plasmid led to a progressive loss of anti-LANA reactivity (Fig. 3, $B$ and $C$ ). Preadsorption with sufficient amounts of orf 73-containing sonicate resulted in the complete loss of the LANA pattern (Fig. $3 \mathrm{D}$ ), indicating that orf 73 protein is the dominant or sole component of LANA.

We also compared the prevalence of antibodies to the recombinant orf 73 gene product with that found for antibodies to classical LANA in the sera from three groups of subjects with differing epidemiologic risks for developing KS. These groups consisted of (i) 50 eligible blood donors, (ii) $33 \mathrm{KS}$ patients, and (iii) 18 women without history of sexual intercourse and no serologic evidence of sexually transmitted diseases (the results of the LANA IFA on these patients have been reported previously [13]). As shown in Table I, parallel IFAs with these sera using both pcDNA3-orf 73 transfected COS7 cells and isolated BCBL-1 nuclei demonstrated a high degree of concordance. Lastly, in individual sera we found that the titer of antibodies to the orf 73 protein expressed in the transiently transfected COS7 cells was indistinguishable from that measured against authentic LANA in BCBL-1 nuclei (data not shown).

\section{Discussion}

By screening for regions of the genome with elevated levels of mRNA expression in uninduced BCBL-1 cells, we identified a 

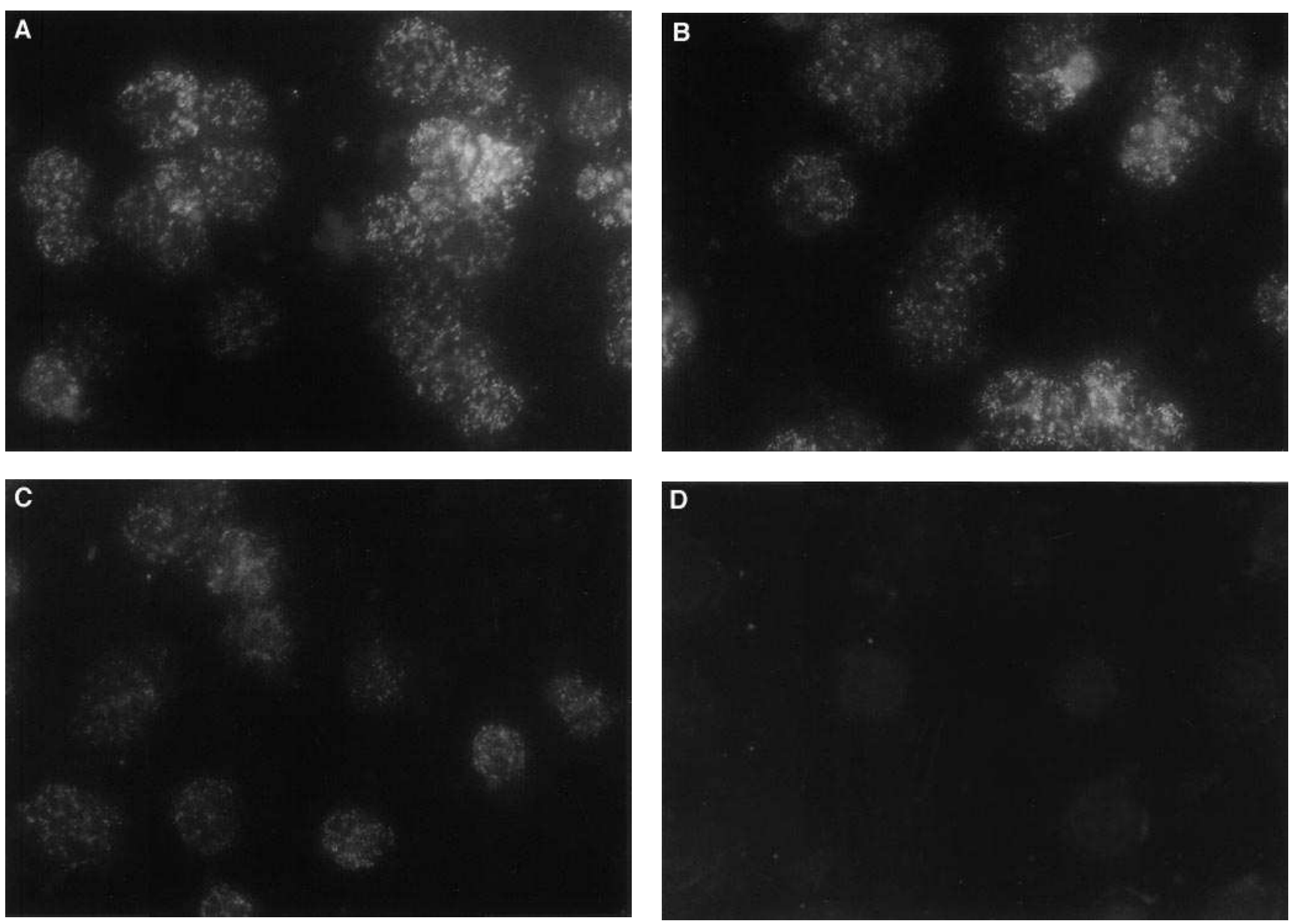

Figure 3. Orf 73 containing COS7 cell sonicates block KSHV (anti-LANA) antibodies. A KS patient serum with an anti-LANA IFA titer of over 1:3,200 was diluted to 1:200 and preadsorbed with sonicates from COS7 cells transfected with vector alone $(A)$; a mixture of sonicates from COS7 cells transfected with either pcDNA3-orf 73 or pcDNA3 in a ratio of 1:3 $(B)$ or 1:1 $(C)$. Preadsorption with sonicate exclusively from cells transfected with pcDNA3-orf 73 is shown in $(D)$. The total protein from sonicates added in $(A-D)$ was identical.

locus bearing several genes whose transcriptional pattern strongly suggests that they encode latent gene products. In Epstein-Barr virus infection, latently expressed viral genes play several key roles, including maintenance of the viral episomal replicon (a function central to viral persistence) and transactivation of a number of host and viral genes involved in the deregulation of cellular physiology and growth (21). Like LANA of KSHV, several of these EBV proteins were initially recognized as nuclear antigens reactive with patient antisera in immunofluorescence assays. In view of these precedents, and since anti-LANA antibodies provide the first visualization of a latent KSHV gene product, the molecular identification of LANA is of some importance.

Our data demonstrate that LANA is comprised predominantly of a single viral protein, encoded by orf 73 of the KSHV genome. Expression of this gene in transient assays results in accumulation of a nuclear antigen reactive with anti-LANA antisera, and authentic anti-LANA reactivity can be completely adsorbed by cell extracts bearing orf 73 as their sole viral protein. In addition, the pattern of serologic reactivity to recombinant orf 73 protein in several patient groups closely mirrors that observed in the canonical LANA assay, leaving no doubt about the assignment of the orf 73 product as the antigen responsible for LANA reactivity. The pattern of antibody staining of recombinant orf 73 shows minor differences in the size and number of the punctate speckles from that observed in LANA expressed in latently infected B cells. These differences may be due, in part, to the overexpression of the orf 73 protein, an interpretation supported by the fact that punctate staining is more evident in COS7 cells expressing lower levels of the protein. However, we do not exclude the possibility that other host or viral proteins could influence the intranuclear distribution of orf 73 protein.

The identification of orf 73 as LANA opens the way to further explorations of its function(s) in the latent infection. Examination of the predicted primary amino acid sequence of the orf 73 product reveal it to be a large polypeptide with numerous repeated blocks of acidic residues; similar domains often function in transcriptional activation in other viral and host proteins (28). In addition, the protein contains sequences compatible with potential coiled-coil domains, elements known to mediate homo- and heterooligomerization. Both of these features are found in proteins involved in the regulation of gene expression, and recall similar features of EBNA-2, a major la- 
Table I.

\begin{tabular}{lrrrrr}
\hline \multicolumn{1}{c}{ Group } & $\begin{array}{c}\text { BCBL-1 } \\
\text { (LANA) }\end{array}$ & COS7-orf 73 & Concordance \\
\hline & & & & & $\%$ \\
& + & - & + & - & \\
Low risk women (18) & 0 & 18 & 0 & 18 & 100 \\
Blood donors (50) & 0 & 50 & 0 & 50 & 100 \\
KS patients (33) & 29 & 4 & 29 & 4 & 100 \\
\end{tabular}

Concordance of LANA (BCBL-1 nuclei) and orf 73 protein (COS7 cells) IFAs in the detection of KSHV antibodies in different populations. Numbers in parentheses are the total number of subjects in each group. The positive $(+)$ and negative ( - ) signs signify the results of the IFAs. All sera were diluted 1:10.

tent protein in EBV. However, we note that orf 73 bears no primary amino acid sequence homology to EBNA 2 or any other known polypeptide.

Finally, the identification of orf 73 protein as LANA makes possible second generation antibody tests using recombinant antigens. While our results (Table I) suggest that second generation immunofluorescence assays with recombinant orf 73 will not outperform the standard anti-LANA test, more sensitive assay formats (e.g., RIA or EIA) may well prove superior and are now within reach.

Note added in proof: Subsequent to the submission of this manuscript, Rainbow et al. have published the finding that orf 73 encodes a high molecular weight protein doublet that is present in another KSHV-infected cell line and that reacts with KS patient sera in both Western analyses and indirect immunofluorescent assays (29).

\section{Acknowledgments}

We thank Dirk Dittmer for helpful discussions.

D. Kedes is supported in part by Universitywide AIDS Research Program award No. R96-SF-142. R. Renne is a special fellow of the Leukemia Society of America. D. Ganem is an investigator of the Howard Hughes Medical Institute.

\section{References}

1. Ambroziak, J., D. Blackbourn, B. Herndier, R. Glogan, J. Gullet, A. McDonald, E. Lennette, and J. Levy. 1995. Herpesvirus-like sequences in HIVinfected and uninfected Kaposi's sarcoma patients. Science (Wash. DC). 268: $582-583$.

2. Boshoff, C., T. Schulz, M. Kennedy, A. Graham, C. Fisher, A. Thomas, J. McGee, R. Weiss, and J. O'Leary. 1995. Kaposi's sarcoma-associated herpesvirus infects endothelial and spindle cells. Nat. Med. 1:1274-1278.

3. Moore, P., and Y. Chang. 1995. Detection of herpesvirus-like DNA sequences in Kaposi's sarcoma lesions from persons with and without HIV infection. N. Engl. J. Med. 332:1181-1185.

4. Schalling, M., M. Ekman, E. Kaaya, A. Linde, and P. Bieberfeld. 1995. A role for a new herpesvirus (KSHV) in different forms of Kaposi's sarcoma. Nat. Med. 1:707-708.

5. Chuck, S., R. Grant, E. Katongole-Mbidde, M. Conant, and D. Ganem. 1996. Frequent presence of a novel herpesvirus genome in lesions of human immunodeficiency virus-negative Kaposi's sarcoma. J. Infect. Dis. 173:248-251.

6. Chang, Y., J. Ziegler, H. Wabinga, E. Katangole-Mbidde, C. Boshoff, T.F. Schulz, D. Whitby, D. Maddalena, H.W. Jaffe, R.A. Weiss, and P.S. Moore. 1996. Kaposi's sarcoma-associated herpesvirus DNA sequences are present in African endemic and AIDS-associated Kaposi's sarcoma. Arch. Intern. Med. 156:202-204.

7. Cesarman, E., Y. Chang, P. Moore, J. Said, and D. Knowles. 1995. Ka- posi's sarcoma-associated herpesvirus-like DNA sequences in AIDS-related body-cavity-based lymphomas. N. Engl. J. Med. 332:1186-1191.

8. Soulier, J., L. Grollet, E. Oksenhendler, P. Cacoub, D. Cazals-Hatem, P. Babinet, M. d'Agay, J. Clauvel, M. Raohael, L. Degos, and F. Sigaux. 1995. Kaposi's sarcoma-associated herpesvirus-like DNA sequences in multicentric castleman's disease. Blood. 86:1276-1280.

9. Whitby, D., M. Howard, M. Tenent-Flowers, N. Brink, A. Copas, C. Boshoff, T. Hatzioannou, F. Suggett, D. Aldam, A. Denton, et al. 1995. Detection of Kaposi's sarcoma associated herpesvirus in peripheral blood of HIVinfected individuals and progression to Kaposi's sarcoma. Lancet. 346:799-802.

10. Jones, J.L., D.L. Hanson, S.Y. Chu, J.W. Ward, and W.H. Jaffe. 1995. AIDS-associated Kaposi's sarcoma. Science (Wash. DC). 267:1078-1079.

11. Gao, S., L. Kingsley, T. Spira, C. Rinaldo, A. Saah, J. Phair, R. Detels, P. Parry, Y. Chang, and P. Moore. 1996. Seroconversion to antibodies against Kaposi's sarcoma-associated herpesvirus-related antigens prior to onset of Kaposi's sarcoma. N. Engl. J. Med. 335:223-241.

12. Moore, P.S., L.A. Kingsley, S.D. Holmberg, T. Spira, P. Gupta, D.R. Hoover, J.P. Parry, L. Conley, H.W. Jaffe, and Y. Chang. 1996. KSHV infection prior to onset of Kaposi's sarcoma. AIDS. 10:175-180.

13. Kedes, D., E. Operskalski, M. Busch, R. Kohn, J. Flood, and D. Ganem. 1996. The seroepidemiology of human herpesvirus 8 (Kaposi's sarcoma-associated herpesvirus): distribution of infection in KS risk groups and evidence for sexual transmission. Nat. Med. 2:918-924.

14. Gao, S.-J., L. Kingsley, M. Li, W. Zheng, C. Parravicini, J. Ziegler, R. Newton, C.R. Rinaldo, A. Saah, J. Phair, et al. 1996. KSHV antibodies among Americans, Italians and Ugandans with and without Kaposi's sarcoma. Nat. Med. 2:925-928.

15. Lennette, E.T., D.J. Blackbourn, and J.A. Levy. 1996. Antibodies to human herpesvirus type 8 in the general population and in Kaposi's sarcoma patients. Lancet. 348:858-861.

16. Simpson, G.R., T.F. Schulz, D. Whitby, P.M. Cook, C. Boshoff, L. Rainbow, M.R. Howard, S.-J. Gao, R.A. Bohenzky, P. Simmonds, et al. 1996. Prevalence of Kaposi's sarcoma associated herpesvirus infection measured by antibodies to recombinant capsid protein and latent immunofluorescence antigen. Lancet. 348:1133-1138.

17. Kedes, D.H., D. Ganem, N. Ameli, P. Bacchetti, and R. Greenblatt. 1997. The prevalence of serum antibody to human herpesvirus 8 (Kaposi sarcoma-associated herpesvirus) among HIV-seropositive and high-risk HIV-seronegative women. J. Am. Med. Assoc. 277:478-481.

18. Goedert, J., D. Kedes, and D. Ganem. 1997. Antibodies to human herpesvirus 8 in women and infants born in Haiti and the USA. Lancet. 349:1368.

19. Beral, V., T. Peterman, R. Berlelman, and H. Jaffe. 1990. Kaposi's sarcoma among persons with AIDS: a sexually transmitted infection? Lancet. 335: $123-127$.

20. Beral, V. 1991. Epidemiology of Kaposi's sarcoma. In Cancer, HIV and AIDS. Vol. 10. V. Beral, H.W. Jaffe, and R. Weiss, editors. Cold Spring Harbor Press, Cold Spring Harbor, NY. 5-22.

21. Kieff, E. 1996. Epstein-Barr virus and its replication. In Fields Virology. Vol. 2. B.N. Fields, D.M. Knipe, P.M. Howley, R.M. Chanock, J.L. Melnick, T.P. Monath, and B. Roizman, editors. Lippincott-Raven, Philadelphia. 2343 2396.

22. Renne, R., W. Zhong, B. Herndier, M. McGrath, N. Abbey, D. Kedes, and D. Ganem. 1996. Lytic growth of Kaposi's sarcoma-associated herpesvirus (human herpesvirus 8) in culture. Nat. Med. 2:342-346.

23. Lagunoff, M., and D. Ganem. 1997. Organization of the termini of the genome of Kaposi's sarcoma-associated herpesvirus (human herpesvirus 8). $\mathrm{Vi}$ rology. 236:147-154.

24. Zhong, W., H. Wang, B. Herndier, and D. Ganem. 1996. Restricted expression of Kaposi's sarcoma-associated herpesvirus genes in Kaposi's sarcoma. Proc. Natl. Acad. Sci. USA. 93:6641-6646.

25. Ausubel, F.M., R. Brent, R.E. Kingston, D.D. Moore, J.G. Seidman, J.A. Smith, and K. Struhl. 1987. Current Protocols in Molecular Biology. Vol. 1. D. Janssen, editor. John Wiley \& Sons, Inc., New York. Suppl. 17:9.11.99.11.10.

26. O’Neill, E., J.L. Douglas, M.L. Chien, and J.V. Garcia. 1997. Open reading frame 26 of human herpesvirus 8 encodes a tetradecanoyl phorbol acetateand butyrate-inducible 32-kilodalton protein expressed in a body cavity-based lymphoma cell line. J. Virol. 71:4791-4797.

27. Russo, J.J., R.A. Bohenzky, M.C. Chien, J. Chen, M. Yan, D. Maddalena, J.P. Parry, D. Peruzzi, I.S. Edelman, Y. Chang, et al. 1996. Nucleotide sequence of the Kaposi sarcoma-associated herpesvirus (HHV8). Proc. Natl. Acad. Sci. USA. 93:14862-14867.

28. Struhl, K. 1995. Yeast transcriptional regulatory mechanisms. Ann. Rev. Genet. 29:651-674.

29. Rainbow, L., G.M. Platt, G.R. Simpson, R. Sarid, S.-J. Gao, H. Stoiber, C.S. Herrington, P.S. Moore, and T.F. Schulz. 1997. The 222- to 234-kilodalton latent nuclear (LNA) of Kaposi's sarcoma-associated herpesvirus (human herpesvirus 8 ) is encoded by orf73 and is a component of the latency-associated nuclear antigen. J. Virol. 71:5915-5921. 